Pre-schoolers' Reasoning in a False-Belief Test Involving Negative Desires

John Bell

\author{
A thesis \\ submitted to Victoria University of Wellington \\ in fulfilment of the requirements for the degree of \\ Masters of Science in Psychology
}

Victoria University of Wellington 


\begin{abstract}
How can people balance competing demands for efficiency and cognitive flexibility in understanding others' beliefs and desires? The idea that humans have two systems for mindreading, a flexible but cognitively demanding system and a minimal, efficient system which operates quickly but possesses signature blindspots was tested. Spontaneous anticipatory looking (AL) and direct verbal predictions of 3- and 4- year-olds were assessed. Children's AL responses displayed a signature blindspot to an agent's desire to avoid an object in an unexpected-transfer false belief task. A dissociation between 4-year-olds' spontaneous AL responses and direct verbal predictions in an avoidance task further supports a 2-mindreading-systems account. The quick efficient mindreading system tracks an agent's desire to approach but not to avoid an object.
\end{abstract}


Acknowledgments

Firstly, I would like to acknowledge and thank my supervisor, Dr. Jason Low for his guidance and encouragement throughout this project.

My sincere appreciation and thanks also go to all the young people who participated in this study, as well as their parents, caregivers and kindergartens. Particular thanks also to all of the kindergarten staff who facilitated data collection and were unfailingly kind and helpful. To Sam, for her constant support and encouragement.

Last, but not least, to my family and friends for their support and love. 
Table of Contents

$\begin{array}{ll}\text { Abstract } & 2\end{array}$

Acknowledgments 3

Table of Contents 4

List of Figures $\quad 5$

$\begin{array}{ll}\text { Introduction } & 6\end{array}$

$\begin{array}{lr}\text { Full (Early-developing) ToM Account } & 8\end{array}$

Minimal-ToM (2-systems) Account 14

$\begin{array}{lr}\text { Approach and Avoidance } & 18\end{array}$

$\begin{array}{ll}\text { The Current Study } & 21\end{array}$

$\begin{array}{ll}\text { Method } & 21\end{array}$

$\begin{array}{ll}\text { Participants } & 21\end{array}$

$\begin{array}{ll}\text { Materials } & 22\end{array}$

$\begin{array}{ll}\text { Procedure } & 22\end{array}$

$\begin{array}{ll}\text { Results } & 28\end{array}$

$\begin{array}{ll}\text { Discussion } & 31\end{array}$

$\begin{array}{ll}\text { References } & 38\end{array}$

$\begin{array}{ll}\text { Appendix } & 42\end{array}$ 


\section{List of Figures}

Figure 1: Familiarisation and belief-induction trials in the approach (positive desire)

false-belief task.

Figure 2: Familiarisation and belief-induction trials in the avoidance (negative desire) false-belief task.

Figure 3: Percentage of participants showing accurate first-look anticipations, and accurate predictions on the think and action questions by age group (3- and 4year-olds) and condition (negative vs. positive desires) 


\section{Pre-schoolers' Reasoning in a False-Belief Test Involving Negative Desires}

The understanding that an agent's internal mental states (thoughts, beliefs, and desires) are influenced by the agent's perception of the environment, and in turn dictate that agent's likely actions, is a hallmark of the possession of a theory of mind (ToM). A theory of mind helps people to understand and negotiate their social world. ToM research was stimulated by the publication of the paper "Does the Chimpanzee Have a Theory of Mind?" (Premack \& Woodruff, 1978). In a commentary on this paper, Dennett (1978) offered a methodological solution that has come to be known as the false-belief task. An observer and an agent observe a scenario. The observer observes a change in the environment which an agent is unaware of. Thus the agent holds a false-belief about the environment. The observer must infer the agent's mistaken belief in order to correctly predict the agent's action.

Following on from Dennett's (1978) suggestions, Wimmer and Perner (1983) investigated young children's understanding of false-belief. They told children a story about a child named Maxi. Maxi puts some chocolate in a cupboard, and goes outside to play. Whilst Maxi is outside, his mother takes the chocolate from the cupboard in order to use some in her baking. Then, rather than returning the chocolate to the cupboard, she places it into a drawer. Maxi's mother leaves the scene. Maxi returns and children were asked where Maxi would look for the chocolate. In order to answer correctly, children must understand that Maxi holds a false belief about the location of the chocolate - Maxi believes the chocolate is in the cupboard where he left it, rather than in its current location, the drawer. This type of task, in which an object is unexpectedly moved from one location to another, is often called an unexpected transfer task. Another version of the false-belief task involves objects with unexpected contents. Unexpected contents tasks can be used to demonstrate to the child that they themselves can hold a false-belief about a situation (Hogrefe, Wimmer, \& Perner, 1986; Perner, Leekam, \& Wimmer, 1987). In such tasks, children are shown a familiar container, 
for example a container of a brand of popular sweets, and asked what is in the package. Typically, children name the popular sweets. The package is then opened to reveal unexpected contents, for example a pencil. Children are then asked what another person who is shown the container will think is inside. Gopnik and Astington (1988) showed children objects which appeared to be different from what they really were - a container of candies which did not contain candy, and a sponge that looked like a rock. Even when children had recent experience of holding a false-belief themselves, their performance was similar to that seen with unexpected transfer tasks.

Wellman, Cross, and Watson (2001) examined a large proportion of the ToM research published prior to January 1998. They performed a meta-analysis of 591 conditions in 178 studies reported in 77 papers or reports, utilising the proportion of false-belief questions answered correctly as the dependent variable. They found that children 3 years and 5 months of age or younger performed at a below chance level. Of note, these young children made a characteristic error in unexpected transfer tasks - they predicted that the agent would search for the object they desired in the location that the object currently was, rather than where the agent believed it to be. Performance on false-belief tasks improves rapidly with age from 3 years and 5 months of age onward. At about four years of age children answer false belief questions correctly at a level above chance.

Wellman, Cross, and Watson (2001) also found that methodological variants of the standard false-belief task that attempt to elevate young children's performance-emphasizing the deliberate attempt to deceive the agent in the scenario, having participants transform or relocate the object in the scenario, removing the target object from the scene when the test question was asked, emphasizing the mental state of the agent — had little effect. One variable, the presence of a temporal marker in the question, was found to interact with the age of children assessed. A temporal marker in a question served to emphasise the time frame 
involved in the scenario (e.g., asking 'where will the agent look first for the chocolate?') The presence of a temporal marker improved performance in children four years and older, but did not improve performance in children younger than four years. However, younger children were not helped by the presence of a temporal marker. Wellman et al. argued that their metaanalysis supported the long-standing developmental view which held that ToM research findings reflect a discontinuous change in children's ToM understanding occurring at approximately four years of age in a typically developing child.

\section{Full (Early-Developing) ToM Account}

Some researchers believe that the demands of the classic false-belief task-requiring verbal ability, the ability to inhibit prepotent responses which assume that a person's belief is true, and working memory to hold relevant aspects of the scenario in mind-obscure possible evidence of full-blown ToM understanding in younger children (e.g., Baillargeon, Scott, \& He, 2010; Bloom \& German, 2000; Leslie \& Polizzi, 1998). They argue that it is possible that young children have developed a theory of mind, or perhaps possess an innate and earlydeveloping understanding of ToM, but that the standard false-belief task is not a sufficiently precise tool to reveal this understanding in younger children. The failure of younger children on false-belief tasks may result from limited information processing ability rather than limited understanding of belief. In an attempt to reduce some of the additional demands placed upon children in false-belief tasks, experimental paradigms were devised to assess false-belief ToM understanding without requiring explicit responses from younger children.

Clements and Perner (1994) were the first to investigate whether young children showed implicit understanding of ToM prior to the development of explicit understanding. In an unexpected-transfer false-belief task, children's eye gazing was videotaped and analysed to see where they looked in anticipation of an agent's actions. They showed children a scenario in which a mouse could exit its house by one of two tunnels. A piece of cheese was 
placed by the mouse in a box next to one of the tunnels. That mouse then went inside his house for a rest. Whilst resting, another mouse came and moved the cheese to a second box adjacent to the other tunnel exit. Waking from his rest, the first mouse felt hungry, and stated that he would go and get his cheese. The experimenter then triggered children's anticipatory looking by wondering aloud where the mouse would look for his cheese. It was found that children aged from 2 years and 5 months of age to 2 years and 10 months of age looked at the present location of the cheese, even though the mouse had not witnessed the object's transfer from the location where he had placed his cheese, and thus held a false-belief about its location. However, about $90 \%$ of children from 2 years and 11 months to 4 years and 5 months of age looked towards the now-empty location where the mouse falsely-believed the object to be. Children were also asked for their explicit prediction of where the mouse would look for the cheese. Only $45 \%$ of the children in the older age bracket, $90 \%$ of whom had looked in anticipation at the now-empty location, were able to explicitly answer that the mouse would look for the cheese in the now-empty box where he had put it before going to sleep. Clements and Perner carefully and modestly interpreted the dissociation between gaze anticipations and verbal predictions as demonstrating that young children possess an implicit understanding of ToM which precedes the development of explicit understanding. Furthermore, they suggested that this implicit understanding develops abruptly at about 2 years and 11 months of age.

Ruffman, Garnham, Import, and Connolly (2001) sought to clarify the phenomena of 3-year-olds giving incorrect explicit responses whilst simultaneously showing correct eye gaze. They gave children ten counters, and asked them to 'bet' the counters on which location they thought a story protagonist would go to. When children bet correctly, the experimenter would double the number of counters bet. When they bet incorrectly, the counters bet would be lost. Betting counters is an explicit measure of ToM, but the measure is not dichotomous - 
children have the opportunity to indicate the confidence with which they understand the situation through betting a proportion of their counters on each outcome, indicating lesser or greater certainty. Children could bet a few of their counters on a second location if they thought that the agent in the story might go to a certain location. Children were tested with both a true- and false-belief task in which a protagonist's return to one of two possible locations was anticipated. Direction of eye gaze and explicit verbal predictions were recorded, and children were asked to put their counters next to the location that they believed the agent would return to, or if they were unsure, to split their counters between the two possible locations. Children showed correct anticipatory looking (AL) at a younger age than they were able to make correct explicit predictions. In the true belief task, children of all ages bet nine or more of their counters on the true belief location. However, in the false-belief task, a different pattern of results was obtained. Younger children who demonstrated correct anticipatory looking but incorrect verbal predictions were very confident about their incorrect verbal prediction, with almost all of these children betting ten counters on the incorrect location. Young children were genuinely not conscious of their anticipatory responses. Older children who failed with explicit predictions were slightly less confident than the younger children, but still bet on average about eight of their counters on the incorrect location. Younger children who passed the explicit verbal prediction question were the least certain of their knowledge, betting on average only about six counters on the correct location. Older children who passed the explicit question were more confident, betting on average eight of their ten counters on the correct location. Children who failed with explicit predictions on the false-belief task were the most confident in their answers. Younger children who passed the false-belief task were the least confident in their knowledge, but gains in confidence were seen with increasing age. Ruffman et al. carefully suggested that the differences between implicit and explicit responses reflected children's possession of different kinds of 
knowledge. Anticipatory looking requires only a representation of a fact, whereas explicit responses require making a judgement about that fact.

Southgate, Senju, and Csibra (2007) investigated 2-year-old children's anticipatory looking using an ingenious non-verbal paradigm. They familiarised children to a situation where an agent reached through one of two windows into a box to get a desired toy. Just prior to the agent reaching through the window, a light would flash and a chime would sound. After two familiarisation trials, children looked in anticipation towards one of the two locations when the light and sound cue occurred. The false-belief scene involved a puppet bear moving a desired toy whilst the agent was distracted. The bear removed the toy from the scene entirely, so that if children were using a rule such as 'ignorance leads to error', then random anticipatory looking should be expected, as both locations were empty, and thus incorrect. Children's eye gaze was analysed. It was found that children reliably looked in anticipation to the location that they would expect the agent to reach to if they attributed a false-belief to the agent. Adopting a rich interpretation, Southgate et al. argued that this showed that 2-year-olds already possessed core ToM understanding, and that this was inconsistent with accounts which posited a conceptual change in children's understanding of false-belief at 4 years of age. They suggested that Clements and Perner's (1994) results, in which they failed to find evidence of implicit ToM understanding in children younger than two years and eleven months of age, may have been affected by the use of the word 'where' in the prompt. Specifically, they suggested that young children may prematurely interpret the word 'where' in the question 'where will the person look for their toy?' as referring to the location in which the toy is currently, rather than the agent's (false) belief about the toy.

The case against conceptual change, however, was famously buoyed by Onishi and Baillargeon's (2005) report of false-belief ToM understanding in 15-month-old infants. They developed a false-belief task which did not require children to use verbal skills in order to 
respond. Using an unexpected-transfer task, they showed infants scenarios in which an actor held a false-belief, and recorded the duration of children's eye gazing. They found that children as young as 15 months looked longer at scenarios in which the agent acted in violation of her false-belief, reaching for a desired toy in a location to which the toy had been moved without the agent's knowledge, rather than reaching for the toy in the location where the agent left it. Onishi and Baillargeon interpreted these results as evidence that 15 -montholds possessed a representational theory of mind, albeit possibly only in an implicit form.

Onishi and Baillargeon's (2005) interpretation of their results are controversial and have been challenged. Perner and Ruffman (2005) suggested three other possible explanations for the results found which did not require possession of a theory of mind. One possible explanation was that the looking times may reflect an association bias - that the children looked longer at new combinations of agent-object-location associations in the test phase as they had not seen them before, and thus more time was needed for cognitive processing. Another possible explanation was that children may believe that ignorance on the part of an agent leads to errors in their actions. The third possibility raised was that children deployed rules about how people behave to understand others' actions (e.g., people look for objects where they last saw them), and the longer looking times reflect children's surprise at the violation of behaviour rules.

Ruffman, Taumoepeau, and Perkins (2012) argued that all ToM tasks confound mental state understanding and knowledge of behaviour. They noted that children's learning abilities are underlain by domain-general statistical learning abilities. Ruffman et al. argued that children's social experiences in the world guide them in generating predictions about the likelihood of given behaviours in given circumstances, and that it is possible that the violation of these probabilistic expectations about how people or agents will behave is responsible for longer looking times in implicit ToM paradigms. They note that early insights 
into people's likely behaviours in a sense implicitly tap mental state understanding because they are based on causal relationships between people and their environment which are underpinned by mental states, but this does not mean that children have knowledge of mental states. They suggest that one way in which children learn about mental states is through parents' use of mental-state terms with their children in everyday conversations, driving children to relate such terms to their own inner experiences. Parents may also use the same mental-state term across a number of different contexts, providing a rich database for children to infer what the underlying similarity in the different contexts is likely to be. Children may also use their experience of the probability of different behaviours being enacted, rather than a more rigid deployment of rules about behaviour, to make sense of their environment and the actions of agents within that environment.

The behaviour rules/probabilities interpretation has not proved easy to rule out, and there is an on-going debate between advocates of the 'behaviour-rules' account and advocates of the 'early-ToM-understanding' account. Proponents of early ToM understanding continue to insist that innate sensitivity to beliefs is a more parsimonious explanation for infants' looking behaviour (Baillargeon, Scott, \& He, 2010; He, Bolz, \& Baillargeon, 2011; Scott \& Baillargeon, 2009) than the behaviour-rules explanation, which requires that children have a rule for a multitude of different scenarios. Proponents of a behaviour rules explanation have replied that behaviour-rule following is more parsimonious, because it does not require the additional step of inferring an intervening mental state between situation and action (Low \& Perner, 2012; Low \& Wang, 2011; Perner, 2010; Wang, Low, Jing, \& Qinghua, 2012). Ultimately, appeals to parsimony are not sufficient to settle the question of how differences between children's spontaneous looking responses and children's direct verbal responses are to be regarded. 


\section{Minimal-ToM (2-systems) Account}

Apperly and Butterfill (2009) suggested that differences between children's implicit and explicit responses to ToM tasks may reflect the possession of not one but two mindreading systems (i.e. a 2-systems account). The two systems allow for a balance to be reached between the competing demands of flexibility and efficiency when predicting others' behaviour. For example, a quick and efficient ToM system would be useful when attempting to predict others' actions in very short time periods, as might occur when a player fakes a pass to deceive their opponent whilst playing a sport. In this situation, reasoning must be quick and efficient in order to respond appropriately to the other player's subsequent actions. When adults engage in speculation or debate about the reason(s) for another person's action(s), such as might occur, for example, in a courtroom setting, they utilise flexible but more time-consuming and cognitively demanding ToM reasoning. This flexible reasoning includes but is not limited to: (a) the representation and negotiation of complex causal reasoning in which an agent's mental states (beliefs, desires, perceptions) lead them to assume intentions, which in turn guide the agent's actions - with these mental states potentially interacting in coherent, yet essentially unbounded ways; (b) the utilisation of abductive reasoning - inference to the best explanation - when there are few restrictions as to what can be inferred to comprise relevant evidence in relation to another person or agent's belief; (c) reporting explanations for others' behaviour which comprise propositional attitudes and a that-clause sentence (e.g., 'the person believes that...') (Apperly \& Butterfill, 2009). The contradictory demands of efficiency and flexibility on cognition and time suggest that exploration of a 2-systems account may prove fruitful.

Under the 2-systems account, a flexible mindreading system underlies explicit verbal reasoning. This system ascribes desires and beliefs to other people and emerges from about 4years of age. Its emergence is scaffolded by development of language, executive function, 
and meta-representational abilities (Apperly, 2011; Low, 2010; Wang et al., 2012).

Anticipatory looking responses are guided by an efficient implicit mindreading system that is shared by infants, children and adults; however, this system is limited in being set to only compute simple relations between objects and agents. These low-level ascriptions involve, but need not be restricted to, tracking an agent's encountering and registration of objects as causally guiding any actions that an agent undertakes in relation to the object (Butterfill \& Apperly, 2013). An agent encounters an object when it enters his sensory field, and registers an object as being in the location where it was recently encountered. Unless the agent subsequently encounters the object in a different location, he will act as if the object is in the location where he has registered it as being. Keeping track of whether an agent encountered an object, and whether or not his registration of the object is correct at the current time, serve as efficient proxies for desire and belief respectively, without requiring the cognitive demands of representing mental states as such. Tracking registration as a belief-like state may explain infant's spontaneous responses in some false-belief tasks (e.g., Onishi \& Baillargeon, 2005) which involve relationships between objects, locations, and agents. The 2 -systems account is predicated on the suggestion that flexibility in representing propositional attitudes such as desires and beliefs is sacrificed in order to achieve cognitive economy in the minimal system.

Apperly and Butterfill's (2009) 2-systems account also hypothesises that the minimal efficient mindreading system is limited in respect of content and psychological role. One of the ways in which the minimal system's limitations in regard to content may manifest is in relation to the identity of an object. The minimal system may not be able to represent different ways of thinking about the same object. Low and Watts (2013) illustrated this point by contrasting a full-blown belief and a minimal-ToM account of a scenario in which an agent has a dual identity. In the world of DC Comics, the most famous inhabitant of the 
fictional city of Metropolis is the superhero Superman, whose alter-ego is Clark Kent. Clark's colleague Lois Lane is unaware that Clark is Superman. If (1) Lois believes that Clark is in a restaurant and (2) Clark is Superman, should it follow that (3) Lois believes that Superman is in the restaurant? In a belief account, the inference that Lois believes Superman is in the restaurant is invalid: Lois does not know that Clark is Superman. Under a minimal-ToM system, where an agent's registrations are tracked, the scenario is as follows: (1) Lois registers Clark-in-restaurant; (2) Clark is Superman; (3) Lois registers Superman-inrestaurant. As registration is a relation to objects rather than to beliefs (Butterfill \& Apperly, 2013), the inference is valid without ascribing a belief to Lois. Someone who represents Lois registering Clark-in-restaurant will not understand why Lois would look elsewhere if searching for Superman, as the registration of Clark-in-restaurant is equivalent to the registration of Superman-in-restaurant. A person using the more flexible but cognitively demanding system for representing beliefs as such would understand that it is possible to think about a single person (Clark) in different ways (Superman), and so may understand why Lois, despite knowing where Clark is, might look elsewhere when searching for Superman.

Low and Watts (2013) investigated signature limits to efficiently tracking registration as a belief-like state in relation to children and adults' implicit responses to an object-identity false-belief task. Three- and four-year-old children and adults watched an identity task which took place in two phases. In the familiarisation phase, people saw two boxes sitting on a table. The boxes lifted, and it was revealed that underneath the left-side box was a red toy, and underneath the right-side box, a blue toy. An agent then entered the scene and observed each toy travel to the opposite box. Windows above the boxes lit up as a chime sounded, and the agent reached for the box in which the blue toy was located. The purpose of the familiarisation phase was to inform the study participants that the agent in the scenario has a preference for one colour over another, in this case for blue over red. In the second, belief- 
induction phase, participants watched the agent observe a toy that appeared to the participants to be a red toy travel from the left- to the right-side box. Out of view of the agent, but visible to the participants, the toy spun around to reveal that its other side was coloured blue. The toy then returned to the left-side box, this time with its red aspect facing the agent and blue aspect facing the study participants. If the study participants are spontaneously making attributions about the agent's belief as such, they should understand that the agent believes that a blue toy remained in the right-side box, and a red toy was located in the left-side box. The accuracy of participants' implicit eye gaze responses was assessed, and explicit verbal predictions were elicited as to which box the agent would reach to. An unexpected-transfer false-belief task was also administered to the same children to assess their implicit eye gaze responses to tracking agent-object relations. Children and adults displayed correct anticipatory looking on the object-location task, but not on the object-identity task. Children's explicit responses showed a developing flexibility with age. On the location test, $31 \%$ of 3 -year-olds, $75 \%$ of 4 year-olds, and $100 \%$ of adults made correct verbal predictions. On the identity test, $13 \%$ of 3 year-olds, $56 \%$ of 4 -year-olds, and $95 \%$ of adults made correct verbal predictions. The pattern of young children's implicit eye gaze allowing them to respond correctly to an object location false-belief but not an object identity false-belief tasks fits with the expectation that the minimal mindreading system has signature limits.

A person's belief is not the only driving force behind their behaviour. They must also hold a desire to achieve a certain state of affairs. Beliefs and desires interact to explain thoughts and actions (Apperly, 2011). In the classic paradigm of the object-location falsebelief task, Maxi desires to find the chocolate. This desire for the chocolate, combined with Maxi's false-belief about the chocolate's location, gives him a subjective reason for looking in the cupboard where he believes the chocolate to be. Given that desires and beliefs play interdependent roles in driving and justifying an agent's actions, and that the demands upon 
cognition required by mindreading attitudes such as intentions and desires are analogous to those required for mindreading beliefs, Apperly and Butterfill (2009) suggested that the minimal efficient mindreading system may also be limited in respect of its ability to represent psychological roles. Thus, a person who only tracks beliefs by processing registrations may not appreciate a situation in which an agent wishes to avoid rather than approach an object. If children's indirect implicit responses, when compared with their explicit understanding, also turn out to show limits on understanding how beliefs can interact with desires, then it can be concluded that the minimal system involves a form of cognition that does not rely upon features of full-blown belief representation as compared to the flexible system.

\section{Approach and Avoidance}

Traditional false-belief tasks utilised an approach paradigm - the agent invariably desires an object. Cassidy (1998) investigated whether 3- to 5-year-old children take an agent's desire into account when reasoning about ToM. She utilised both approach (the agent wants the object) and avoidance (the agent does not want the object) scenarios. Children were introduced to a puppet and shown three different types of food - the puppet's favourite food (desired), a food the puppet thought was 'ok' (neutral food), and a food that the puppet hated to eat (not desired). Two unexpected transfer scenarios were then shown. Children were told they would play a game with the puppet. The puppet had to hide its food in one of two hiding places. Whichever hiding place the puppet chose, it would have to eat the food in that location. In one trial, the puppet took their favourite food and hid it in one of two hiding places. The puppet left the room, and the experimenter and child participant moved the puppet's favourite food to the other location. The puppet returned, and the child was asked which hiding place the puppet would pick. In the second trial, the process was repeated, only this time with the food the puppet hated. Pilot work had shown children disliked pointing to an empty box. For this reason, on both trials the neutral food was placed with the desired or 
undesired food in the initial location and not moved, meaning that when children were asked to choose a location both locations had food in them. Cassidy found that 4-year-old children who successfully passed the false-belief task in the desired-food scenario did not perform better than chance in the undesired food scenario, despite correctly identifying the location the undesired food was in. Cassidy suggested one possible explanation for the results obtained was that the necessity of combining the calculation of the agent's false belief and the calculation of the behaviour necessary to satisfy the desire to avoid the disliked food overwhelmed children's cognitive resources.

Building on Cassidy's (1998) work, Leslie and Polizzi (1998) suggested that the differing explicit prediction performances of three- and four-year-old children in false-belief tasks may result from differences in executive functioning abilities - in particular, differences in the ability to inhibit prepotent responses. They suggested that people have a default attribution - that the other person holds a true-belief - in regard to other people's belief(s). In order to demonstrate understanding of a false-belief, the person being assessed must first inhibit their initial default response, based on the assumption that the other person's belief is true, in order to search for an alternative explanation for the other person's actions - in this case, that the other person holds a false-belief. This view assumes that failure to successfully inhibit the initial assumption that the other person has a true-belief is responsible for young children's poor explicit prediction performance on false-belief tasks. They further suggested that in order to understand an avoidance paradigm (in which the agent does not want an object, but in effect desires to avoid the location in which the object is present), a person must first attend to the location of the object to be avoided, and then inhibit that location and switch their attention to the second location, where the object to be avoided is not located. Thus a successful explicit prediction of an agent's actions in an avoidance paradigm in a false-belief task requires a double inhibition, the inhibition of the assumption of true-belief, 
and the inhibition of the location where the object to be avoided is currently, in order to select the location where the object is not. Leslie and Polizzi (1998) noted that the two inhibitions cannot simply be summed, as this would produce the incorrect answer. Rather, the two inhibitions must interact in order to cancel each other out. Thus a task involving double inhibition would be considerably more difficult than a task involving single inhibition.

To investigate their hypothesis about the role of inhibition in ToM understanding, Leslie and Polizzi (1998) utilised an avoidance paradigm in conjunction with a false-belief unexpected-transfer task. They tested 4-year-olds who had succeeded in a false-belief task in an approach scenario. Children were presented with a scenario in which a girl, Renee, does not want to put a piece of fish in the same box as her sick pet kitten, who is resting in one of two boxes in the scene. In the false-belief condition the sick kitten crawls from one box to the other whilst Renee is absent retrieving the piece of fish. In the true-belief condition, Renee retrieves the piece of fish and returns to witness the kitten crawl from one box to the other. Upon Renee's return, children were asked where Renee thinks (false-belief) or knows (truebelief) the kitten is, and to predict which box Renee will go to with the fish. The think/know question required that children consider only Renee's belief. The prediction question required that children consider both Renee's belief and her (negative) desire (to avoid placing the fish in the same box as the kitten). $6 \%$ of children in the true belief and $11 \%$ of children in the false-belief condition failed the think/know question which required a single inhibition. A double inhibition was required to successfully answer the prediction question, which required children to also take into account Renee's (negative) desire to avoid placing the piece of fish in the same box as the sick kitten. When a false-belief was coupled with a negative desire, four-year-olds performed poorly. $62 \%$ of children failed the prediction question. Leslie and Polizzi noted that this was a considerably larger failure rate than would be expected if a double inhibition was simply the sum of two single inhibitions, in which case a failure rate of 
$17 \%$ would have been expected. They concluded that the findings supported their inhibition hypothesis, and thus supported the information processing account of differences in threeand four-year-olds performance in false-belief tasks - that earlier possession of understanding of ToM is masked in younger children by executive function demands.

\section{The Current Study}

The current study utilises an approach and an avoidance scenario to assess young children's implicit and explicit ToM understanding in a false-belief unexpected-transfer task. An agent wants to put away his toy rabbit, and has a choice of two boxes, one of which already contains a toy snake. In the positive-desire or approach scenario, the agent wants to place the rabbit in the box with the snake, as the two toys are friends. In the negative-desire or avoidance scenario, the agent does not want to put the rabbit in the box which the snake is in, as the rabbit is afraid of the snake. The agent holds a false-belief about the location of the snake, which has been unexpectedly transferred to the other box whilst the agent was distracted. The full (early-developing) ToM account would suggest a certain pattern of results - that implicit responses would be accurate across approach and avoidance scenarios. Following Low and Watts' (2013) minimal (2-systems) ToM account, it is hypothesised that young children's implicit understanding of ToM would allow them to track facts about other people approaching objects, but would not allow them to track facts about other people avoiding objects. Thus, it is expected that children's anticipatory eye gazing will be accurate in approach scenarios, but not in avoidance scenarios.

\section{Method}

\section{Participants}

Forty 3-year-olds (25 males, 15 females; $M=42.63$ months, $S D=3.35$, age range $=$ 36-47 months) and forty 4-year-olds (22 males, 18 females; $M=54.25$ months; $S D=3.30$, age range $=49-59$ months) from six local kindergartens participated in the study. Information 
letters and consent forms (Appendix A) were sent to the parents of all children at the participating kindergartens. Informed parental consent was obtained and only those children with parental consent were invited to take part. Children also provided assent prior to taking part. Ethics approval for the study was granted by the School of Psychology Human Ethics Committee under delegated authority from the Victoria University of Wellington's Human Ethics Committee. Participants spoke English and were interviewed individually at their kindergarten. Forty participants were randomly assigned to the approach (positive-desire) condition and forty participants to the avoidance (negative-desire) condition.

\section{Materials}

The approach and avoidance false-belief unexpected-transfer tasks were recorded in digital video format and edited before being shown as a film to participants on a 22 -inch television monitor. A video camera was used to record the direction of participants' eyegazing. A remote control was used to start and pause the film as required. In the films an agent wearing a plain blue shirt and a white visor sat behind two tables which were arranged so that a small gap ran between them from left to right across the scene. On the table, located to either side of the agent, were two boxes (one blue, one green, with brown lids). Both boxes opened on the side closest to the agent, allowing him to reach inside. A black panel with strop light sat on top of each box. A telephone was situated on a stand behind the agent. The toys used in the familiarisation phases were a small multi-coloured rugby ball, a toy car, a toy train and a toy teapot. In the test phase, the toys used were a white rabbit hand-puppet, a pink pig hand-puppet and a plush green toy snake.

\section{Procedure}

Each participant watched a film of either an approach or an avoidance scenario on a 22-inch television monitor in a quiet room at their local kindergarten. Each scenario comprised two familiarisation trials and one belief-induction trial. Participants were 
interviewed individually by an experimenter. A high-definition video camera placed behind and above the television monitor was trained on children's faces to record their anticipatory looking.

The approach scenario task comprised two familiarisation trials and one beliefinduction trial (see Figure 1). In the first familiarisation trial, children saw an agent introduce himself, saying "Hi, my name is Jason, and this is my toy". The agent wore a white visor so that his eyes were obscured. He was seated at a desk with a box on either side, holding a toy on the desk in front of him. Then the black panel on top of both boxes flashed with a beep sound. In response to the light and sound cue, the agent said, "that means it's time to put the toy away'. He opened one of two different coloured boxes located to either side of him, and pulled out a toy from inside the box. He said, "There is a toy in this box. Toys should go together." He then replaced the toy he had removed from the box and closed the box, before reaching across to the other box, opening it, and observing aloud said, "And this box is empty". He then closed that box. The light and sound cue occurred again, and the agent said, "That's right, I still have to put the toy away". The agent reached for and opened the first box which had a toy inside, and placed the toy which was sitting on the desk in front of him into the box, which he then closed.

The second familiarisation trial followed the same order of events, but with different toys on the desk in front of the agent and inside the boxes, and with the agent reaching to the opposite box from that he reached for first in the first familiarisation trial. The purpose of the familiarisation trials was to teach children that the light and sound cue signalled to the agent that it was time to put his toy away. All participants showed correct first look anticipations in the second familiarisation trial. Since participants were familiar with the cue, it should trigger their anticipatory looking (AL) in the test trial. 


\section{Familiarisation Trial}

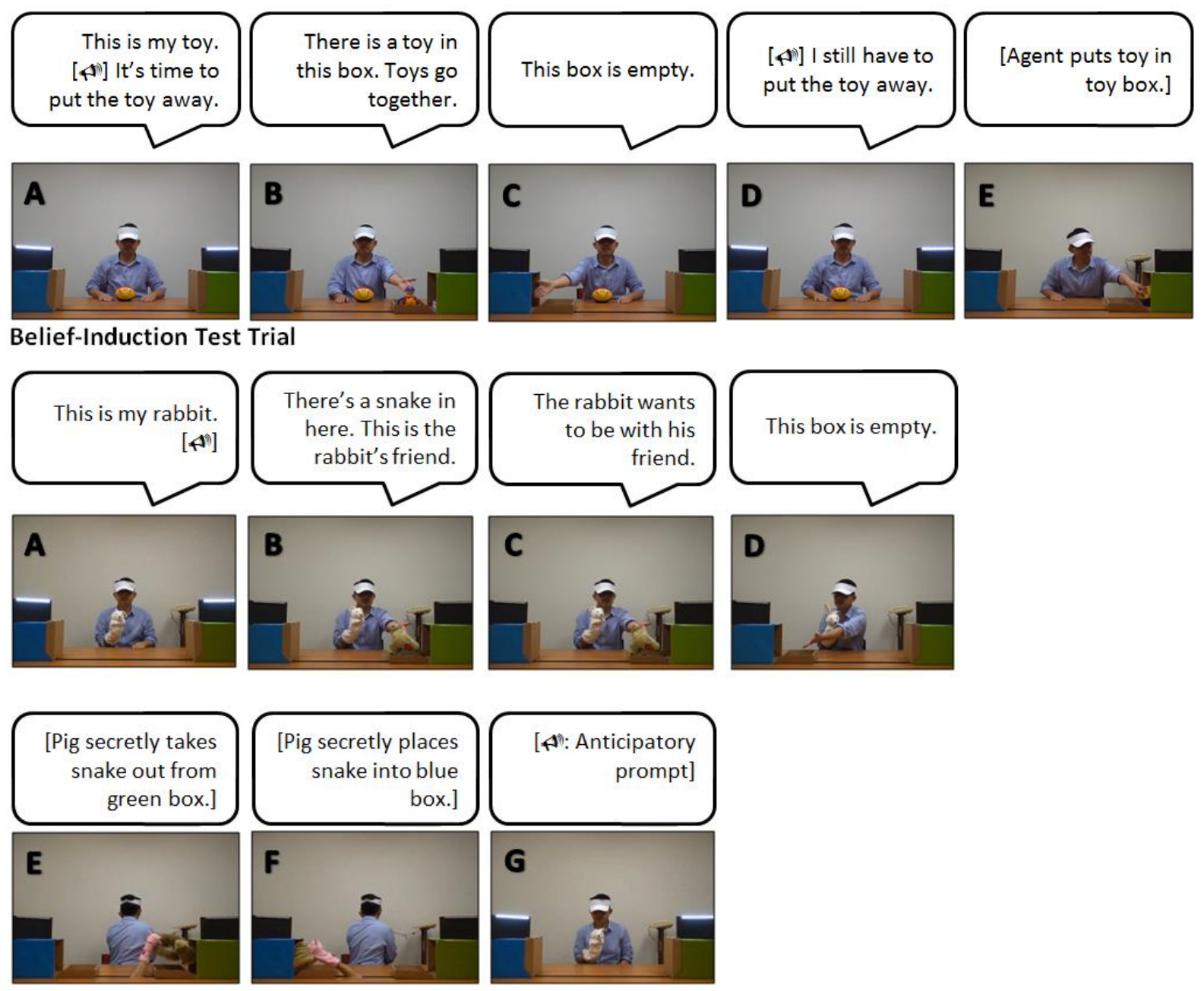

Figure 1. Familiarisation and belief-induction trials in the approach (positive desire) falsebelief task.

The familiarisation trials in the avoidance scenario (see Figure 2) followed a similar procedure to the familiarisation trials in the approach scenario. The scenarios diverged when the agent (the same actor, Jason) opened the first box. He pulled out a toy, said "This box is full", replaced the toy, and closed the box. He then reached across to the other box, opened it, observed "And this box is empty', and then closed that box. The cue occurred, and the agent stated, “That's right, I still have to put the toy away'. He then placed the toy in the empty box. The second familiarisation trial followed the same series of events, but with different toys, and with the empty box located on the opposite side from the first familiarisation trial. 


\section{Familiarisation Trial}

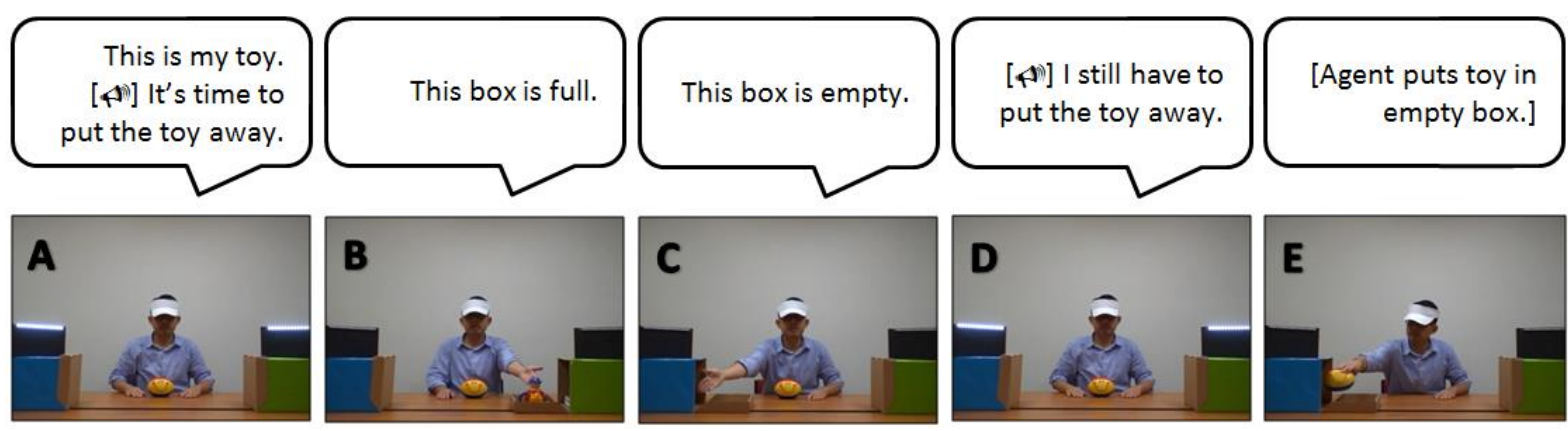

\section{Belief-Induction Test Trial}
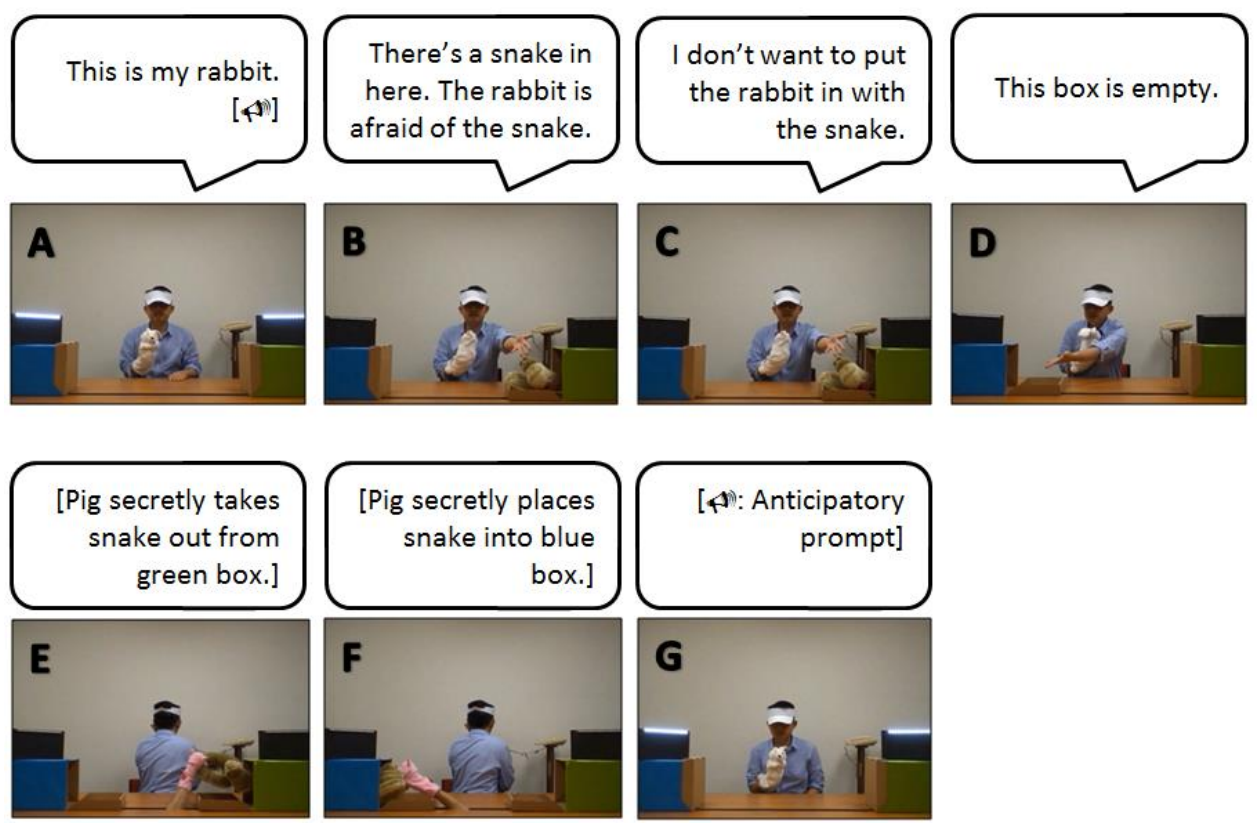

Figure 2. Familiarisation and belief-induction trials in the avoidance (negative desire) falsebelief task.

All participants who participated in the avoidance condition also showed correct first look anticipations in the second familiarisation trial.

Belief-induction trial. A belief-induction test trial followed the familiarisation trials in both conditions (see Figures 1 and 2). This was the important scenario in terms of differentiating the full-ToM account from the minimal-ToM account. Two effectively identical versions of the belief-induction trial were filmed for both the approach and avoidance scenarios allowing for counterbalancing of target locations. The belief-induction trial began with the agent once more seated at the desk, this time holding a hand-puppet 
rabbit on his right hand. The agent re-introduced himself saying ' $\mathrm{Hi}$, my name is Jason, and this is my rabbit' (and the rabbit's head moved from side to side). The light-and-sound cue occurred, and the agent reached for one of the boxes, opened it, and pulled out a toy snake.

Approach (positive-desire) condition. In the approach scenario the agent said 'There's a snake in here. This is the rabbit's friend. The rabbit wants to be with his friend'. As he said 'the rabbit wants to be with his friend', the rabbit's paw was made to wave at the snake. The agent replaced the snake in its box, closed the box, and reached across to the other box and opened it. He noted, "And this box is empty", and then closed the second box. At this point a telephone which was located on a stand behind the agent rang, and the agent turned around so that he was facing away from the desk. The agent picked up the telephone, said hello, and appeared to listen to a conversation. Whilst he was engaged with the telephone and facing away from the scene, a hand-puppet pig rose up through a gap in the middle of the desk. The pig opened the box in which the snake was located, opened the empty box, transferred the snake to the empty box, closed that box, closed the first box, and left the scene. At that point, the agent said "OK, bye", and then hung up the telephone and turned back to face the desk. As such, the agent did not witness the transfer of the snake. The cue then occurred. The critical issue is where children would anticipate the agent reaching towards to put the rabbit away. During the first 1,750-ms following the light-and-sound cue, analyses focused on determining whether participants showed AL to the left- or right-side box (first-look direction and looking duration were measured; Low, 2010; Low \& Watts, 2013).

Avoidance (negative-desire) condition. The avoidance scenario was effectively identical to the approach scenario except as follows. When the agent opened the box in the avoidance scenario, he said, “There's a snake in here! The rabbit is afraid of the snake! I don't want to put the rabbit in with the snake because that will make the rabbit more scared". 
As he said 'the rabbit is afraid of the snake. I don't want to put the rabbit in with the snake because that will make the rabbit more scared' the rabbit was animated such that it turned to face the agent and was visibly shaking and trembling until the agent replaced the snake in its box and closed the box.

Questions. To ensure that the participants followed the scenario, the film was paused at certain moments and each participant was asked control story memory questions. After the agent opened the box and explained that 'the rabbit was friends-with or afraid-of the snake' the experimenter asked, "Does Jason want to put the rabbit in with the snake?". If the participant answered incorrectly the experimenter would correct the child, stating either "Yes - Jason wants to put the rabbit in with the snake because the rabbit and the snake are best friends" (approach condition) or "No - Jason does not want to put the rabbit in with the snake because that will make the rabbit more scared" (avoidance condition). The experimenter then asked the participant to "Point to which box the snake is inside". Again, if the child indicated the incorrect box, the experimenter would correct the child, indicating the correct box. After the unexpected transfer of the snake by the pig, the child was asked to "Point to which box the snake is now inside". If a child answered incorrectly he or she was informed of the correct answer. Only 1 participant (a 3-year-old in the avoidance condition) had to be corrected when answering where the snake (after the unexpected transfer) was located.

At the end of the film, after the 1750-ms AL time-window, children were asked two test questions: a think question "Point to which box Jason thinks the snake is inside" and a prediction question "Point to which box Jason will put the rabbit in".

Coding. Two raters coded $25 \%$ of AL recordings during the first 1,750-ms following the illumination-sound cue in the belief-induction trials for both conditions. The coding was done by playing back each recording in slow-motion and scoring gaze direction on a frameby-frame basis (looking times were accurate to $0.04-\mathrm{s} ; 25$ frames/s) using the Avidemux 2.5 
programme (Low, 2010). Raters agreed $100 \%$ of the time as to whether or not participants looked first and looked more to the correct location. One of the raters then coded the remaining recordings.

\section{Results}

The percentages of participants showing accurate first-look anticipations, and accurate predictions on the think and action questions by age group (3- and 4-year-olds) and condition (negative vs. positive desires) are summarised in Figure 3. Across both the approach and avoidance conditions accurate explicit verbal responses increased as a function of age for both the think and prediction prompts. There were no gender-related effects.

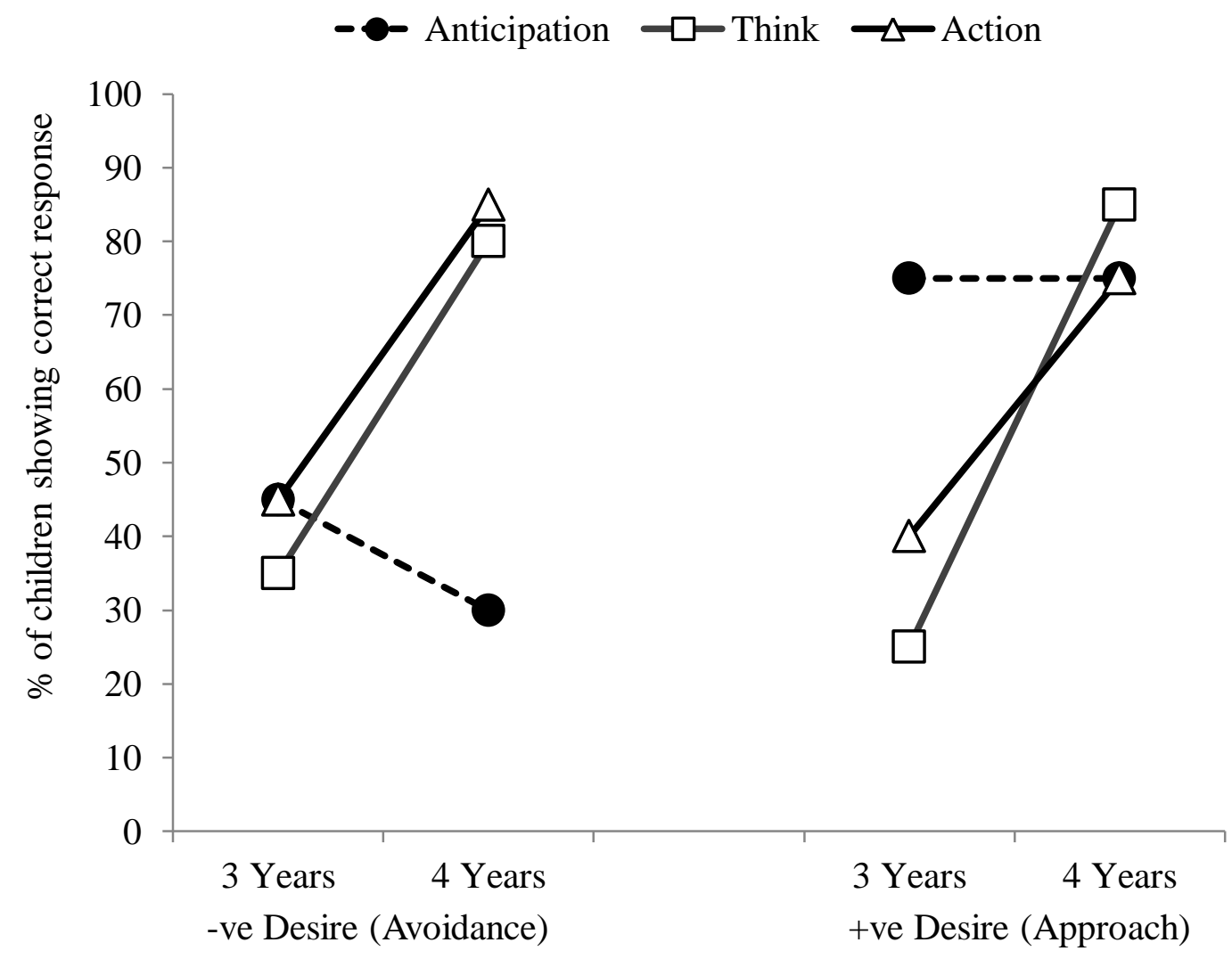

Figure 3. Percentage of participants showing accurate first-look anticipations, and accurate predictions on the think and action questions by age group (3- and 4-year-olds) and condition (negative vs. positive desires). 
In the approach task, 15 of 203 -year-olds (75\%) and 15 of 204 -year-olds (75\%) demonstrated first look anticipations towards the correct location - for each age group, a greater percentage of correct first looks than would be expected by chance (binomial test, $p \mathrm{~s}$ $<.05)$. In the avoidance task, performance in correct anticipatory looking was poorer. Only 9 of 20 3-year-olds (45\%) and 6 of 20 4-year-olds (30\%) demonstrated anticipatory looking toward the correct location in the test trial of the avoidance task, neither better nor worse than would be expected by chance (binomial test, $p \mathrm{~s}>.05$ ).

Duration of anticipatory looking was also analysed — based on a differential looking score - over the 1,750 millisecond window following the light and sound cue in the beliefinduction trial for each participant (Senju, Southgate, White, \& Frith, 2009). The DLS (ranging from -1 to +1 ) is a measure of preference for looking to one target location over another, and was calculated by subtracting total looking time to the incorrect window from total looking time to the correct window and then dividing by the sum of looking time to the correct and incorrect windows. An analysis of variance (ANOVA) was performed on participants' DLS, with the valence of desire (approach condition vs. avoidance condition) and age group (3- vs. 4-year-olds) as between-subjects factors. There was no significant main effect of age group $(F(1,76)=0.49, p>.05)$. There was a significant main effect of valence condition $\left(F(1,76)=17.95, p<.001 ; \eta_{\mathrm{p}}{ }^{2}=.191\right)$. On average, participants looked longer to the correct location in the approach condition and looked longer to the incorrect location in the avoidance condition ( $M=261$-ms vs. $M=-286-\mathrm{ms})$. The age group by valence condition interaction was not significant $(F(1,76)=0.19, p>.05)$.

As indicated in Figure 3, there were age-related changes in 3- and 4-year-olds performances on the explicit prediction test questions. In the avoidance condition 7/20 (35\%) 3-year-olds responded correctly to the think question, and 16/20 (80\%) 4-year-olds responded correctly, $\chi^{2}(1, N=40)=8.29, p<.05$. For the prediction question $9 / 20(45 \%) 3$-year-olds 
responded correctly, and 17/20 4-year-olds responded correctly $(85 \%), \chi^{2}(1, N=40)=7.03, p$ $<.05$. In the approach condition, $25 \%$ of 3 -year-olds responded correctly to the think question, and $85 \%$ of 4 -year-olds responded correctly, $\left.\chi^{2}(1, N=40)=14.55, p<.05\right)$. In response to the prediction question, $40 \%$ of 3 -year-olds made accurate predictions, and $75 \%$ of 4-year-olds made accurate predictions, $\chi^{2}(1, N=40)=5.01, p<.05$.

Across age groups, performances across the think and prediction questions were significantly correlated in the avoidance condition $(r=0.61, p<.001 ; N=40)$ and in the approach condition $(r=0.34, p<.05 ; N=40)$. Significant inter-relationships between performance on the think and prediction questions remained in the avoidance condition even when individual differences associated with age were removed $\left(r_{\text {partial }}=0.56, p<.001, d f=\right.$ 37). There was no significant age-partialled correlation between children's performance in the think and prediction questions in the approach condition $\left(r_{\text {partial }}=0.16, p>.05, d f=37\right)$.

Three-year-olds' anticipatory first looks and verbal predictions in the avoidance condition were not significantly associated for either the think prompt (Wilcoxon $Z=-0.632$, $p>.05$ ) or the prediction prompt (Wilcoxon $Z=0.00, p>.05$ ). However, four year olds displayed a significant dissociation between anticipatory looking and verbal predictions in the avoidance condition for both the think prompt and the prediction prompt. For the think prompt, $55 \%$ of 4 -year-olds gave accurate verbal predictions despite anticipatory looking to the incorrect side (Wilcoxon $Z=-2.887, p<.01$ ). For the prediction prompt, $60 \%$ of 4 -yearolds gave accurate verbal predictions despite anticipatory looking to the incorrect side (Wilcoxon $Z=-3.051, p<.01$ ).

The analyses then turned to investigate potential dissociations between the accuracy of participants' first look and the accuracy of participants' predictions on the think and prediction questions. For 3-year-olds in the avoidance condition, there were no significant dissociations between first look anticipations and direct verbal responses on the prediction 
question, and no significant dissociations between first look anticipations and direct verbal responses on the think question (Wilcoxon $\mathrm{Z}$ test, all $p \mathrm{~s}>.05$ ). However, 3-year-olds showed significant dissociations in their patterns of responding in the approach condition. A significant number of 3 -year-olds $(60 \% ; 12 / 20)$ showed the classic dissociation pattern of displaying accurate first looks despite giving inaccurate answers to the think question (Wilcoxon $\mathrm{Z}=-2.67, p<.01)$. Similarly, $45 \%$ of 3 -year-olds $(9 / 20)$ also showed the classic dissociation pattern of displaying accurate first looks despite giving inaccurate answers to the prediction question (Wilcoxon $\mathrm{Z}=-2.11, p<.05$ ).

With respect to the avoidance condition, 4-year-olds showed a reverse pattern of dissociation. A significant number of 4-year-olds $(12 / 20 ; 60 \%)$ displayed incorrect first looks while providing accurate verbal responses to the prediction question (Wilcoxon $\mathrm{Z}=-3.06, p$ $<.01)$. Similarly, a significant number of 4-year-olds $(11 / 20 ; 55 \%)$ displayed incorrect first looks while providing accurate verbal responses to the prediction question (Wilcoxon $\mathrm{Z}=$ $2.89, p<.01)$. The 4-year-olds did not show any significant pattern of dissociation in the approach condition (Wilcoxon $\mathrm{Z}$ test, all $p \mathrm{~s}>.05$ ).

\section{Discussion}

The full-and-early-developing ToM account predicted that there should be clear AL to the correct side in both the approach and the avoidance tasks, revealing children's implicit ToM understanding. Following Apperly and Butterfill's (2009) 2-systems account, it was hypothesised that young children's implicit understanding of ToM would allow them to track facts about other people approaching objects, but would not allow them to track facts about other people avoiding objects. Thus, it was expected that children's AL would be accurate in approach scenarios, but not in avoidance scenarios. A clear side preference in AL (as measured by first look direction) was observed in both 3- and 4-year-olds in the approach task. However, no clear side preference in AL (as measured by first look direction) was 
observed in either 3- or 4-year-olds in the avoidance task, supporting Apperly and Butterfill's account. The findings indicated that an efficient mind-reading or minimal-ToM system does not accommodate negative desires ('the agent does not want...'), such as the desire to avoid an object, but rather accommodates only positive desires ('the agent wants...'), such as when an agent holds a desire to approach an object. Three- and 4-year olds were able to, as evidenced by their anticipatory looking (AL), accurately track facts relating to others' beliefs regarding object location in situations where the other person expressed a desire to approach an object. However, in a task in which a person expressed a desire to avoid an object and had a mistaken belief about the object's whereabouts, 3- and 4-year-olds' AL was at chance. AL was accurate in approach scenarios, but in avoidance scenarios AL was at at-chance levels, suggesting that a minimal-ToM system was ineffective when faced with situations involving complex desire and belief interactions. Further mapping on Apperly and Butterfill's 2systems view, explicit verbal predictions developed in step, showing an age-related increase in accuracy across both the approach and avoidance tasks. This suggests that explicit predictions are guided by a separate flexible mind-reading system. Four-year-olds were successful with explicit predictions across both the approach and avoidance tasks, whilst 3 year-olds were unsuccessful. Overall, the results support the existence of a minimal-ToM ability as hypothesised by a 2-systems account, whilst simultaneously failing to support the pattern of results predicted by the full-ToM account.

These results complement those found by Low and Watts (2013) in their investigation of the signature limitations hypothesised to form part of a minimal-ToM system in a 2systems account of ToM development. They found that AL was not accurate in a task where it was necessary to compute how an agent would misinterpret an identity of an agent, where the truth of the agent's beliefs would result in there being more than one object in the scene (in reality there was only a single object; the object had a different colour on each side, a fact 
which was known to the test participants but not to the agent in the test scenario). Children's and adults' implicit understanding allowed them to track facts involving object location but not object identity. Low and Watts concluded that both children and adults possess a minimal-ToM system which is not capable of taking object identity into account. The results of the current study suggest that the minimal-ToM system is even more rudimentary - it is not capable of considering how desires can interact complexly with beliefs. As such, the minimal ability to track registration as belief-like state appears to be limited to tracking an agent's desire to approach an object and fails to handle an agent's negative desire, such as the desire to avoid an object.

A single-system or full-ToM account might explain the current study's results by claiming that the avoidance task involves a greater degree of executive ability: more information processing demand may be required to calculate a negative desire as opposed to a positive desire. Crucially, 4-year-olds' explicit responses in the avoidance task were correct, despite their AL responses showing no preference for either the correct or incorrect location. Moreover, single-system accounts (Baillargeon, Scott, \& He, 2010) assume that explicit but not implicit responses require the deployment of executive abilities. For this reason the earlydeveloping (innate) ToM account fails to predict the pattern of results obtained. If spontaneous looking responses are a more accurate and more sensitive manifestation of an innate ToM understanding, children (4-year-olds) should not be showing superior performance in their direct verbal responses as compared to their indirect AL responses.

Leslie and Polizzi (1998) granted a pivotal role to the process of inhibition in children's early-developing ToM reasoning. They theorised that reasoning about negative desires in a false-belief task required a double inhibition - an inhibition of a default assumption of true-belief and an inhibition of the location where the non-desired object is which would be significantly more taxing on executive abilities than either inhibition alone. 
They presented two possible models of inhibitory processing in belief problems, both of which relied upon the subject mustering two inhibitions in order to make an accurate explicit prediction. In either model, failure of any of the inhibitory processes results in error. The think question (asked of children in the current study) required only a single inhibition - that of an assumption of true-belief - whereas the prediction question required the 'double inhibition' of both belief and location. Thus, Leslie and Polizzi’s double inhibition theory predicts that children's performance would be (considerably) worse on the prediction than the think question. However, in the current study 4-year-olds correctly answered both the think ( $80 \%$ correct) and prediction ( $85 \%$ correct) questions in the avoidance task, with slightly better performance on the prediction question. When 4-year-olds were able to correctly answer a question about the agent's (false) belief, they were also able to predict the agent's action, even when the agent had a negative desire.

Four-year-olds' explicit predictions in the avoidance task in the current study were accurate. These results differ from those obtained by Cassidy (1998) and Leslie and Polizzi (1998), who found that 4-year-olds did not pass similar false-belief avoidance tasks. Why did 4-year-olds pass the avoidance task in the current study whilst 4-year-olds in previous studies failed explicit prediction questions? One obvious answer is that the methodology of the current study differed from that of both Cassidy's and Leslie and Polizzi's studies. The current study used edited films to show children in each condition the same sequence of events. Previous studies manually re-enacted the storyline for each child, which is likely to have resulted in greater variability in the presentation of the task. Using an edited film allowed for the relevant aspects of the scene to be presented in a visually salient manner. Cassidy's task required that the child remember which of three foods was desirable, neutral, and undesirable in the opinion of the agent. Leslie and Polizzi's task required that children recall that it was detrimental to feed fish to the sick kitten - that the preferred food, 
unusually, causes illness. Children may have had to inhibit the idea that food is beneficial. Steps were taken in the current study to ensure that children understood key aspects of the storyline through the use of control story memory questions posed at key moments. These questions may have assisted children by emphasising key aspects of the scenario, decreasing children's reliance upon memory. They also served to highlight the reason for the agent's desire, which children may have found helpful. In the avoidance task in the current study, the rabbit trembled at the sight of the snake, highlighting its fear. However, it is also possible that young children may also lack experience in viewing complex ToM scenarios and thus fail to pay attention to aspects of the story (such as complex belief and desire interactions) that older viewers pay particular attention to. It may also be possible that 4-year-olds' success in the false-belief avoidance task in the current study reflects the fact that children at this age are capable of directly reasoning about a negative-desire false-belief task when other demands upon executive abilities are reduced.

Perner and Ruffman (2005) suggested a behaviour-rule account to explain young children's accurate looking responses (and perhaps explicit predictions). A behaviour-rule account explains children's accurate AL as resulting from the deployment of rules that are constructed from inferences based on past experiences. A behaviour rule such as 'when putting toys away, people choose a location (box) where they have seen other toys' could underlie children's AL responses in the approach task. However, the lack of a clear side preference in the avoidance task for either 3- or 4-year-olds is not easily explained by a behaviour rules account. That said, children may be less likely to generate a behaviour rule for a situation where a person wants to avoid an object. Ruffman, Taumoepeau, and Perkins (2012) suggested that children may also use their experience of the probability of different behaviours being enacted, rather than a more rigid deployment of rules about behaviour, to make sense of their environment and the actions of agents within that environment. Rule- 
based inference about a positive desire can be made whenever a person moves to approach an object. In the context of object avoidance, there is no clear spatial link may between a person and the object he or she wishes to avoid, and thus rules may not be so easily inferred from such experiences. However, as for the Behaviour Rules explanation, the use of probabilistic reasoning cannot explain the pattern of results obtained, specifically why 4 -year-olds in the avoidance task displayed incorrect AL and correct explicit predictions. Probabilistic reasoning should lead to either consistent success or failure across AL and explicit predictions, or to a pattern of correct AL and incorrect explicit predictions. Overall, though, children's social-learning experiences, and the inferences about probabilities that children may make as a result, could still contribute to the basis of a minimal-ToM system.

What further evidence would strengthen the case for a signature limitation to AL with respect to tracking negative desires? It is important to note that the current study only tested 3- and 4-year-old children, in whom understanding of false-belief is still developing. Further research should investigate whether people in other age groups also fail to display accurate AL in false-belief avoidance tasks. Apperly, Warren, Andrews, Grant, and Todd (2011) have already investigated the accuracy and speed of 6- to 11-year-old children and adults' explicit responses to belief-desire reasoning tasks. They found that although accuracy and speed improved with increasing age, false-belief negative desire tasks remain more difficult for older children and adults than other combinations of belief and desire. This remained the case even when participants were explicitly told whether the agent had a true or false-belief, obviating the need to infer the agent's belief. The minimal-ToM hypothesis predicts that the same signature limitations in AL accuracy observed in 3- and 4-year-old children will also be observed in older children and adults.

Another way that the evidence for a minimal-ToM system could be strengthened would be to demonstrate the existence of signature limits within-subjects. Thus, the same 
participants could be tested on both a positive- and a negative-desire scenario. If accurate AL was observed in the positive-desire scenario but not in the negative-desire scenario consistently within-subjects, that would strengthen the case for a minimal-ToM system. Similarly, experiments employing a within-subjects design to reveal the extent to which individual children and adults show limits in implicit (AL) understanding of both false-beliefs over object identity and negative desires would further bolster the evidence for a minimalToM system and the 2-systems account.

The current study demonstrates that AL is not accurate in scenarios in which an agent holds a negative desire, a desire to avoid rather than approach an object. This adds to the body of evidence which suggests that the ToM system evidenced by children's AL has signature limitations, consistent with the 2-systems account proposed by Apperly and Butterfill (2009). Two ToM systems, one a minimal system which tracks agent-object relations, and the other a more complex system which is able to reason about propositional attitudes, provides the best solution to the competing demands for efficiency and flexibility in ToM tasks. 


\section{References}

Apperly, I. A. (2011). Mindreaders: The cognitive basis of "Theory of Mind". Hove and New York: Psychology Press.

Apperly, I. A., \& Butterfill, S. A. (2009). Do humans have two systems to track beliefs and belief-like states? Psychological Review, 116(4), 953-970. doi: 10.1037/a0016923

Apperly, I. A., Warren, F., Andrews, B. J., Grant, J., \& Todd, S. (2011). Developmental continuity in theory of mind: Speed and accuracy of belief-desire reasoning in children and adults. Child Development, 82(5), 1691-1703. doi: 10.1111/j.14678624.2011.01635.x

Baillargeon, R., Scott, R. M., \& He, Z. (2010). False-belief understanding in infants. Trends in Cognitive Sciences, 14(3), 110-118. doi: 10.1016/j.tics.2009.12.006

Bloom, P., \& German, T. P. (2000). Two reasons to abandon the false belief task as a test of theory of mind. Cognition, 77(1), B25-B31. doi: 10.1016/S0010-0277(00)00096-2

Butterfill, S. A., \& Apperly, I. A. (2013). How to construct a minimal theory of mind. Mind \& Language, 28(5), 606-637. doi: 10.1111/mila.12036

Cassidy, K. W. (1998). Three-and four-year-old children's ability to use desire- and beliefbased reasoning. Cognition, 66(1), B1-B11. doi: 10.1016/S0010-0277(98)00008-0

Clements, W. A., \& Perner, J. (1994). Implicit understanding of belief. Cognitive Development, 9, 377-395. doi: 10.1016/0885-2014(94)90012-4

Dennett, D. C. (1978). Beliefs about beliefs. The Behavioral and Brain Sciences, 1(4), 515526.

Gopnik, A., \& Astington, J. W. (1988). Children's understanding of representational change and its relation to the understanding of false belief and the appearance-reality distinction. Child Development, 59(1), 26-37. doi: 10.2307/1130386 
He, Z., Bolz, M., \& Baillargeon, R. (2011). False-belief understanding in 2.5-year-olds: Evidence from violation-of-expectation change-of-location and unexpected-contents tasks. Developmental Science, 14(2), 292-305. doi: 10.1111/j.14677687.2010.00980.x

Hogrefe, G. J., Wimmer, H., \& Perner, J. (1986). Ignorance versus false belief: A developmental lag in attribution of epistemic states. Child Development, 57(3), 567582. doi: $10.2307 / 1130337$

Leslie, A. M., \& Polizzi, P. (1998). Inhibitory processing in the false belief task: Two conjectures. Developmental Science, 1(2), 247-253.doi: 10.1111/1467-7687.00038

Low, J. (2010). Pre-schoolers' implicit and explicit false-belief understanding: Relations with complex syntactical mastery. Child Development, 81(2), 597-615. doi: 10.1111/j.1467-8624.2009.01418.x

Low J., \& Perner, J. (2012). Implicit and explicit theory of mind: State of the art. British Journal of Developmental Psychology, 30, 1-13. doi: 10.1111/j.2044835X.2011.02074.X

Low, J. \& Wang, B. (2011). On the long road to mentalism in children's spontaneous falsebelief understanding: Are we there yet? Review of Philosophy and Psychology, 2(3), 411-428. doi: 10.1007/s13164-011-0067-y

Low, J., \& Watts, J. (2013). Attributing false beliefs about object identity reveals a signature blind spot in humans' efficient mind-reading system. Psychological Science, 24(3), 305-311. doi: 10.1177/0956797612451469

Onishi, K. H., \& Baillargeon, R. (2005). Do 15-month-old infants understand false beliefs? Science, 308, 255-258. doi: 10.1126/science.1107621

Perner, J. (2010). Who took the cog out of cognitive science? Mentalism in an era of anticognitivism. In P. A. Frensch \& R. Schwarzer (Eds.), International perspectives on 
psychological science: Cognition and neuropsychology (pp. 241-262). New York, NY: Psychology Press.

Perner, J., Leekam, S. R., \& Wimmer, H. (1987). Three-year-olds' difficulty with false belief: The case for a conceptual deficit. British Journal of Developmental Psychology, 5(2), 125-137. doi: 10.1111/j.2044-835X.1987.tb01048.x

Perner, J. \& Ruffman, T. (2005). Infants' insight into the mind: How deep? Science, 308, 214-216. doi: 10.1126/science. 1111656

Premack, D. \& Woodruff, G. (1978). Does the chimpanzee have a theory of mind? The Behavioral and Brain Sciences, 1(4), 568-570.

Ruffman, T., Garnham, W., Import, A., \& Connolly, D. (2001). Does eye gaze indicate implicit knowledge of false belief? Charting transitions in knowledge. Journal of Experimental Child Psychology, 80(3), 201-224. doi: 10.1006/jecp.2001.2633

Ruffman, T., Taumoepeau, M., \& Perkins, C. (2012). Statistical learning as a basis for social understanding in children. British Journal of Developmental Psychology, 30, 87-104. doi: 10.1111/j.2044-835X.2011.02045.X

Scott, R. M., \& Baillargeon, R. (2009). Which penguin is this? Attributing false beliefs about object identity at 18 months. Child Development, 80(4), 1172-1196. doi: $10.1111 / \mathrm{j} .1467-8624.2009 .01324 . x$

Senju, A., Southgate, V., White, S., \& Frith, U. (2009). Mindblind eyes: An absence of spontaneous theory of mind in Asperger syndrome. Science, 325(5942), 883-885. doi: 10.1126/science. 1176170

Southgate, V., Senju, A., \& Csibra, G. (2007). Action anticipation through attribution of false belief by 2-year-olds. Psychological Science, 18(7), 587-592. doi: 10.1111/j.14679280.2007.01944.x 
Wang, B., Low, J., Jing, Z., \& Qinghua, Q. (2012). Chinese preschoolers' implicit and explicit false-belief understanding. British Journal of Developmental Psychology, 30, 123-140. doi: 10.1111/j.2044-835X.2011.02052.x

Wellman, H. M., Cross, D., \& Watson, J. (2001). Meta-analysis of theory-of-mind development: The truth about false belief. Child Development, 72(3), 655-684.

Wimmer, H. \& Perner, J. (1983). Beliefs about beliefs: Representation and constraining function of wrong beliefs in young children's understanding of deception. Cognition, 13(1), 103-128. doi: 10.1016/0010-0277(83)90004-5 
Appendix A

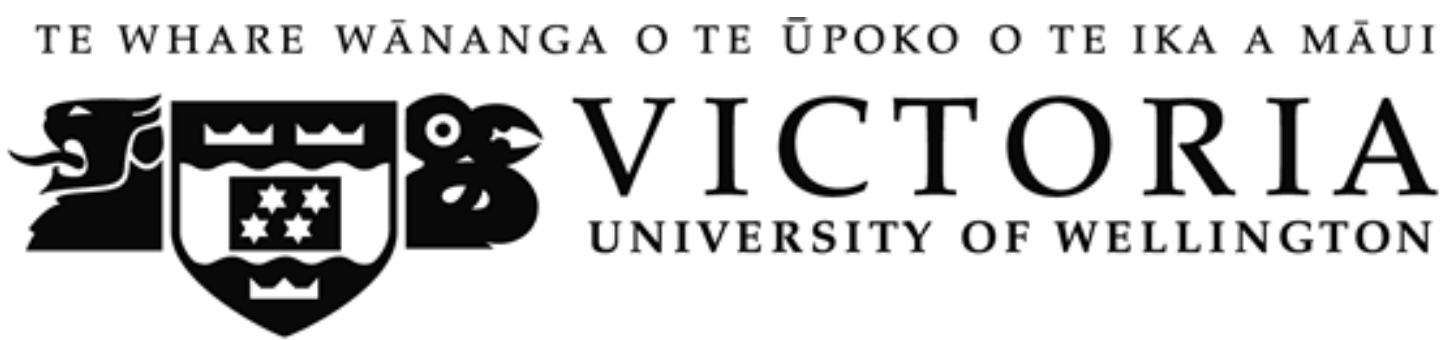

Research Study Information Sheet:

What Young Children Understand about People's Minds

John Bell and Dr Jason Low

School of Psychology, Victoria University of Wellington

\section{What is the purpose of this research?}

- We wish to learn about the age trends by which children come to spontaneously discover that other people can think differently to themselves, and that people's thoughts and beliefs about the world influence behaviour (this understanding is formally known as children's Theory of Mind development). We are inviting 3-year-olds and 4-year-olds to take part in this research project.

\section{Who is conducting the research?}

- John Bell is a student in the Clinical Psychology programme, and Dr Jason Low is a Senior Lecturer in Psychology. John is carrying out the research as part of his Masters thesis project. Dr Low is the project supervisor. This research has been approved by the School of Psychology Human Ethics Committee under delegated authority of Victoria University of Wellington's Human Ethics Committee.

\section{What is involved if you agree to let your child participate?}

- Each child will be invited to watch a short film clip with toy puppets on a TV screen (only around 3-minutes long). The story is about a man wanting to hide a soft-toy rabbit inside a box. In the story, the man sees that the green box is full (it already has a soft-toy snake in it) and that the blue box is suitably empty. The man says he does not want to put the toy rabbit with the toy snake; however whilst the man is distracted, a puppet pig comes along and secretly moves the toy snake from the green box to the blue box. Then the man turns around and wants to hide the toy rabbit. Children will be asked to predict where the man will go to hide the toy rabbit, in the green or blue box. We hope to learn more about the age when children start to spontaneously understand that the man will put the toy rabbit in the blue box because he thinks that box is empty and thinks that the toy snake is in the green box.

- We will also use a camera to video-record children's gazing whilst they are watching the film clips. We wish to learn if children can unconsciously sense the correct answer even if they give an incorrect verbal answer. So in the case of the puppet show, we wish to learn whether children will look first and gaze longer at the blue box if they unconsciously anticipate the man's belief about where to put the toy rabbit.

- This project will help uncover whether young children show an intuitive understanding of people's thoughts and beliefs in their gazing responses compared to their verbal responses.

- As such, the task is straight-forward and will only take around 3-minutes. All testing will take place at your child's school in a quiet space that is designated and seen by your child's teacher. The project will take place at times convenient to your child's teacher.

- You do not have to make any special attempt to get your child to think about such tasks beforehand as it is children's own spontaneous responses that we are interested in. 
- At the end of the study your child will get to collect stickers.

- During the research you are free to withdraw your child from the study, without needing to give a reason, at any point before your child's data have been collected. Your child will also be asked if he or she would like to take part on the day of the study itself. Your child is also free to decline without having to give a reason.

\section{Privacy and Confidentiality}

- Consent forms, video recordings and verbal response sheets will remain in Dr Low's custody and kept in a locked laboratory. Consent forms will be kept separate from all other data. Once we have coded the duration of children's gaze from the video recordings, all video recordings will be wiped. Information from the video recordings and verbal responses will be coded by number only. Your child will not be identified in the research project or in any presentation or publication.

- The coded data may be used in other related studies. Results of this project may be published but any data included will in no way be linked to any individual child. In accordance with the requirements of some scientific journals and organisations, the coded data may be shared with other competent researchers. Again, your child's anonymity will be preserved.

- Consent forms and coded data will be kept for five years after publication.

\section{What happens to the information that you provide?}

- After enough information has been collected, the overall findings may be submitted for publication in a scientific journal, presented at a scientific conference or deposited as a thesis in the Victoria University Library.

- The overall findings may form part a PhD thesis, Masters thesis, or Honours research project that will be submitted for assessment.

- Summary information about the general findings from this study will be sent to your child's school when the study is completed and the analyses of the findings available.

\section{How can you contact us?}

- If you have any further questions regarding this study please do not hesitate to contact John Bell (E-mail: John.Bell@vuw.ac.nz; phone 0276363948) or the project supervisor Dr Jason Low (E-mail: Jason.Low@vuw.ac.nz; phone: 463-6721).

What should you do if you want to give permission for your child to take part?

- Should you give consent for your child to participate, please complete and return the attached Statement of Informed Consent to your child's school teacher as soon as possible.

Whether or not you decide that your child can take part in this research, we would like to thank you for considering this opportunity. Yours Sincerely,
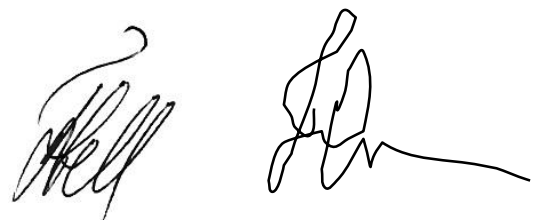

John Bell and Dr Jason Low

School of Psychology, Victoria University of Wellington

PO Box 600, Wellington, New Zealand

E-mail: John.Bell@vuw.ac.nz OR Jason.Low@vuw.ac.nz

Phone: 0276363948 OR 463-6721 


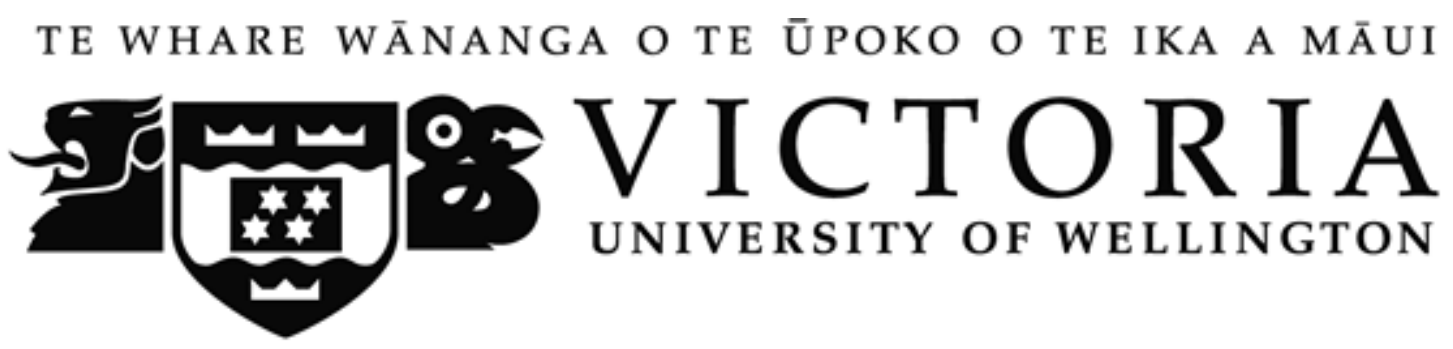

\author{
Research Project: What Young Children Understand about People's Minds \\ John Bell and Dr Jason Low
}

\title{
Consent Form
}

I have read the information about this research and I understand that:

- I can withdraw my child at any time, without needing to give a reason, prior to the end of his or her participation. My child can also refuse to participate.

- My child will be filmed to help learn about children's gaze responses, and film recordings will be wiped after analysis.

- My child will never be identified in the research project or in any other presentation or publication. The information my child provides will be coded by number only.

- All response sheets and coded information will be stored separately from consent forms.

- The data, which the results of the study depend upon, will be treated in strictest confidence.

- The results of the study may be published but my child's anonymity will be preserved.

I agree that my child can participate in this research.

Name of Parent or Guardian:

Signature of Parent or Guardian:

Date of Signature:

Name of Participating Child:

Participating Child's Age:

Years Months

If you would like a summary of findings when it is available, please write your e-mail here: 